\title{
Transverse Shear Viscosity to Entropy Density for the General Anisotropic Black Brane in Horava-Lifshitz Gravity
}

\author{
Mehdi Sadeghi* \\ Department of Physics, School of Sciences, \\ Ayatollah Boroujerdi University, Boroujerd, Iran
}

May 9, 2019

\begin{abstract}
In this paper we calculate the ratio of transverse shear viscosity to entropy density for the general anisotropic black brane in Horava-Lifshitz gravity. There is a wellknown conjecture that states this ratio should be larger than $\frac{1}{4 \pi}$. The ratio of shear viscosity to entropy density is proportional to the inverse square coupling of quantum thermal field theory, $\frac{\eta}{s} \sim \frac{1}{\lambda^{2}}$. Especially in QFT with gravity dual the stronger coupling means the shear viscosity per entropy density gets closer to the lower bound of $\frac{1}{4 \pi}$. The KSS bound preserves in the anisotropic scaling model.
\end{abstract}

PACS numbers: 11.10.Jj, 11.10.Wx, 11.15.Pg, 11.25.Tq

Keywords: Fluid/Gravity duality, AdS/CFT duality, transverse Shear viscosity, GreenKubo formula

\section{Introduction}

The AdS/CFT duality [1, 2, 3] states that quantum gravity theories, such as string theory, on an $A d S_{d+1}$ background is dual to a $d$-dimensional CFT, which is a nongravitational theory. Hydrodynamics [4] is an effective theory of QFT at large distances and time-scales. AdS/CFT duality leads to fluid/gravity duality in this limit [్, 6]. The hydrodynamics equations are laws of conservation of energy and momentum [4, 7],

$$
\begin{aligned}
\nabla_{\mu} T^{\mu \nu} & =0, \\
T^{\mu \nu} & =(\rho+p) u^{\mu} u^{\nu}+p g^{\mu \nu} .
\end{aligned}
$$

*mehdi.sadeghi@abru.ac.ir 
According to dictionary of AdS/CFT, Black Brane within the bulk is dual to a fluid on the boundary .

Table 1: Relevant equations and parameters in the bulk and on the boundary

\begin{tabular}{|c|c|}
\hline Bulk & Boundary \\
\hline$E_{M N}=R_{M N}-\frac{1}{2} R g_{M N}+\Lambda g_{M N}=0$ & $\nabla_{\mu} T^{\mu \nu}=0$ \\
\hline$d s^{2}=-2 u_{\mu} d x^{\mu} d r+r^{2}\left[\eta_{\mu \nu}+\left(\frac{r_{+}}{r}\right)^{4} u_{\mu} u_{\nu}\right] d x^{\mu} d x^{\nu}$ & $T^{\mu \nu}=(\pi T)^{4}\left(g^{\mu \nu}+4 u^{\mu} u^{\nu}\right)$ \\
\hline
\end{tabular}

where $r_{+}, \Lambda, T$ and $u_{\mu}$ are event horizon, cosmological constant, temperature and fluid velocity, respectively [6].

Since the hydrodynamics regime is valid and $\varepsilon=\frac{l_{m f p}}{L}<<1$, we expand it to the leading order of $\varepsilon[5]$.

$$
\begin{aligned}
& T^{\mu \nu}=(\rho+p) u^{\mu} u^{\nu}+p g^{\mu \nu}-\sigma^{\mu \nu}, \\
& \sigma^{\mu \nu}=P^{\mu \alpha} P^{\nu \beta}\left[\eta\left(\nabla_{\alpha} u_{\beta}+\nabla_{\beta} u_{\alpha}\right)+\left(\zeta-\frac{2}{3} \eta\right) g_{\alpha \beta} \nabla . u\right], \\
& P^{\mu \nu}=g^{\mu \nu}+u^{\mu} u^{\nu},
\end{aligned}
$$

where $\eta, \zeta, \sigma^{\mu \nu}$ and $P^{\mu \nu}$ are shear viscosity, bulk viscosity, shear tensor and projection operator, respectively [6, 8, 9, 10]. In this work we are interested in calculating transverse shear viscosity by Green-Kubo formula [10, 11].

$$
\eta=\lim _{\omega \rightarrow 0} \frac{1}{2 \omega} \int d t d \vec{x} e^{\imath \omega t}\left\langle\left[T_{y}^{x}(x), T_{y}^{x}(0)\right]\right\rangle=-\lim _{\omega \rightarrow 0} \frac{1}{\omega} \Im G_{y}^{x} x_{y}^{x}(\omega, \overrightarrow{0}) .
$$

In the following, we consider the general black brane in the Horava-Lifshitz gravity [12, 13, 14, 15, 16]. Then, we calculate the shear viscosity to the entropy density ratio by Green-Kubo formula. The ratio is found to satisfy the conjectured bound $\frac{1}{4 \pi}$ for this gravity.

\section{Anisotropic Black Brane in Horava-Lifshitz Gravity}

We study the model with anisotropic scaling. Horava- lifshitz and Einstein-Hilbert gravity with a scalar field, the so-called khronon [17] have this property. It help us to understand tricritical point in condense matter physics named by Griffiths [18]. Since, Horava-Lifshitz gravity is a well known theory, we don't mention to its details. There are several ways to create this theory: It can be obtained by coupling a massive gauge field to Einstein gravity or as solutions of Einstein-axion-dilaton [13, 12] theories as follows,

$$
I=\int d^{5} x \sqrt{-g}\left(R-2 \Lambda-\frac{1}{2}(\partial \phi)^{2}-\frac{1}{2} e^{2 \phi}(\partial \chi)^{2}\right)
$$


Where $\chi$ and $\phi$ are axion and dilaton fields respectively. The axion field has a constant profile in the radial direction and depends linearly on $\mathrm{z}, \chi=a z$. The metric of nonextreme black brane in the context of HL gravity is,

$$
d s^{2}=-r^{2 \alpha} h(r) d t^{2}+\frac{d r^{2}}{r^{2} f(r)}+\frac{r^{2}}{l^{2}} b(r)\left(d x^{2}+d y^{2}\right)+\frac{r^{2}}{l^{2}} k(r) d z^{2},
$$

Functions $h(r)$ and $f(r)$ are the blackening factors. Metric (7) is invariant under anisotropic scaling of space-time coordinates $t \rightarrow \lambda^{\alpha} t, x_{i} \rightarrow \lambda x_{i}, r \rightarrow \frac{r}{\lambda}$ where $\alpha$ is the dynamical critical exponent.

Since, we are considering non-extreme black holes, both functions have simple root at the horizon $r=r_{+}$, such that $h\left(r_{+}\right) / f\left(r_{+}\right)$is finite at the horizon.

For later convenience, we introduce the dimensionless coordinate $u=\frac{r_{+}^{2}}{r^{2}}$,

$$
d s^{2}=-\frac{r_{+}^{2 \alpha}}{u^{\alpha} l^{2 \alpha}} H(u) d t^{2}+\frac{l^{2} d u^{2}}{4 u^{2} F(u)}+\frac{r_{+}^{2}}{u l^{2}} B(u)\left(d x^{2}+d y^{2}\right)+\frac{r_{+}^{2}}{u l^{2}} K(u) d z^{2}
$$

where $l$ is radius of AdS. The solution is clearly isotropic in the $x y$-directions, but not in the $z$-direction.

The temperature and the Hawking-Bekenstein entropy density read as [19],

$$
\begin{aligned}
T & =\left.\frac{1}{4 \pi \sqrt{g_{u u} g_{t t}}} \partial_{u} g_{t t}\right|_{u=1}=\left.\frac{r_{+}^{\alpha}}{2 \pi l^{\alpha+1}} \sqrt{\frac{F}{H}} H^{\prime}\right|_{u=1}=\left.\frac{r_{+}^{\alpha}}{2 \pi l^{\alpha+1}} \sqrt{F^{\prime} H^{\prime}}\right|_{u=1}, \\
s & =\frac{4 \pi}{V} \int d^{3} x \sqrt{-g}=4 \pi\left(\frac{r_{+}}{l}\right)^{3} B(u=1) \sqrt{K(u=1)}
\end{aligned}
$$

Now to find the transverse shear viscosity, one may consider tensor perturbation of the background metric as $g_{x y}+h_{x y}$ [19, 20, 21] where $x, y$ are parallel to the brane. Since the brane has translational invariance, we apply Fourier transformation.

$$
h_{x}^{y}(t, u, \vec{x})=G(u) \int \frac{d^{4} k}{(2 \pi)^{4}}\left(h^{0}\right)_{x}^{y}(\omega, \vec{k}) \exp (\imath \vec{k} \cdot \vec{x}-\imath \omega t) .
$$

Regarding the Green-Kubo formula it is enough to set $\vec{k}=0$. Plug the perturbed metric in the action of HL gravity and keep terms up to $G^{2}$ :

$$
I_{2}=\int d^{5} x\left(K_{1} G^{2}-K_{2} G^{2}\right)
$$

where

$$
\begin{aligned}
& K_{1}=\sqrt{-g} g^{u u} \\
& K_{2}=\frac{l^{2 \alpha} \sqrt{-g}}{r_{+}^{2 \alpha} u^{-\alpha} H(u)} \omega^{2}
\end{aligned}
$$

then the EoM is,

$$
\left(K_{1} G^{\prime}\right)^{\prime}+K_{2} G=0
$$


Eq.(13) shows that the perturbation satisfies the Klein-Gordon equation as follows,

$$
\frac{1}{\sqrt{-g}} \partial_{u}\left(\sqrt{-g} g^{u u} \partial_{u} h_{x y}(t, u, \vec{x})\right)+g^{\mu \nu} \partial_{\mu} \partial_{\nu} h_{x y}(t, u, \vec{x})=0
$$

By substituting Eq.(11) in Eq.(14)

$$
\frac{1}{\sqrt{-g}} \partial_{u}\left(\sqrt{-g} g^{u u} \partial_{u} G(u)\right)-g^{t t} \omega^{2} G(u)=0,
$$

using (8) we obtain,

$$
G^{\prime \prime}(u)+\frac{1}{2}\left(\frac{F^{\prime}}{F}+\frac{H^{\prime}}{H}+\frac{2 B^{\prime}}{B}+\frac{K^{\prime}}{K}-\frac{\alpha+1}{u}\right) G^{\prime}(u)+\frac{l^{2 \alpha+2} \omega^{2} G(u)}{4 r_{+}^{2 \alpha} u^{2-\alpha} F(u) H(u)}=0,
$$

in which ' denotes derivative with respect to $u$. This equation is singular at the horizon $u=1$. Thus, first of all, we study the near horizon behavior by using the Taylor expansion,

$$
\begin{aligned}
& F(u) \approx-(1-u) F^{\prime}(1), \\
& H(u) \approx-(1-u) H^{\prime}(1) .
\end{aligned}
$$

then,

$$
F(u) H(u) \approx(1-u)^{2} F^{\prime}(1) H^{\prime}(1)=(1-u)^{2}\left(\frac{2 \pi l^{\alpha+1} T}{r_{+}^{\alpha}}\right)^{2} .
$$

Therefore Eq. (16) transforms to,

$$
G^{\prime \prime}(u)-\frac{1}{1-u} G^{\prime}(u)+\frac{\omega^{2}}{16 \pi^{2} T^{2}} \frac{1}{(1-u)^{2}} G(u)=0 .
$$

The solution to the above equation is $G(u)=(1-u)^{\beta}$, with

$$
\beta= \pm \frac{\imath \varpi}{2}, \varpi \equiv \frac{\omega}{2 \pi T}
$$

In the above equation we choose the minus sign, since we are interested in the incoming waves at the horizon. Coming back to the main equation (16), we consider the following ansatz with an expansion in terms of $\varpi$,

$$
G(u)=\widetilde{F}(u)^{\frac{-\imath \varpi}{2}}\left(\widetilde{h}_{0}(u)+\frac{\imath \varpi}{2} \widetilde{h}_{1}(u)+O\left(\varpi^{2}\right)\right),
$$

where $\widetilde{F}(u) \equiv \sqrt{F(u) H(u)}$ and we set $\widetilde{h}_{0}(u)=1$ to normalize $G(u)$ on the boundary. Plugging (20) into (16) to first order of $\varpi$, we obtain,

$$
\widetilde{h}_{1}^{\prime \prime}+\frac{1}{2}\left(\frac{\widetilde{F}^{\prime}}{\widetilde{F}}+\frac{2 B^{\prime}}{B}+\frac{K^{\prime}}{K}-\frac{\alpha+1}{u}\right) \widetilde{h}_{1}^{\prime}-\frac{\widetilde{F}^{\prime \prime}}{\widetilde{F}}+\frac{\widetilde{F}^{\prime}}{2 \widetilde{F}}\left(-\frac{2 B^{\prime}}{B}-\frac{K^{\prime}}{K}+\frac{\alpha+1}{u}\right)=0 .
$$


The solution to the above equation is as follows,

$$
\begin{array}{r}
\frac{\widetilde{F} \widetilde{h}_{1}^{\prime}-\widetilde{F}^{\prime}}{B^{-1} K^{\frac{-1}{2}} u^{\frac{\alpha+1}{2}}}=C_{1}, \\
\widetilde{h}_{1}=\log \frac{\widetilde{F}}{C_{2}}+C_{1} \int^{u} \frac{B^{-1} K^{\frac{-1}{2}} u^{\frac{\alpha+1}{2}}}{\widetilde{F}} d u,
\end{array}
$$

Where $C_{1}$ and $C_{2}$ are integration constants. For our purposes the explicit form of $\widetilde{h}_{1}$ is not important. It would be enough to find $C_{1}$ by demanding $\widetilde{h}_{1}$ to be non-singular at the horizon. So we may investigate the near horizon behavior of the integral in (23)) as follows,

$$
\begin{aligned}
\widetilde{F} & \approx-(1-u) \widetilde{F}^{\prime}(1)=-(1-u) \frac{2 \pi l^{\alpha+1} T}{r_{+}^{\alpha}} \\
\widetilde{h}_{1} & \approx \log \frac{1-u}{C_{2}}-\frac{C_{1} r_{+}^{\alpha}(B(u=1))^{-1}(K(u=1))^{\frac{-1}{2}}}{2 \pi l^{\alpha+1} T} \log (1-u) .
\end{aligned}
$$

Considering a non-singular $\widetilde{h}_{1}$ at the horizon, $C_{1}$ read as ,

$$
C_{1}=\frac{2 \pi l^{\alpha+1} T}{r_{+}^{\alpha}} B(u=1) \sqrt{K(u=1)}
$$
be:

Following the prescription given in [22, 23, 24], the retarded Green's function will

$$
\begin{aligned}
G_{y y}^{x x}(\omega, \overrightarrow{0}) & =-\left.\sqrt{-g} g^{u u} G^{*}(u) \partial_{u} G(u)\right|_{u \rightarrow 0} \\
& =\left.\frac{\imath r_{+}^{\alpha+3} \varpi}{\ell^{\alpha+4}}\left[\frac{\widetilde{F}^{\prime}-\widetilde{F} \widetilde{h}_{1}^{\prime}}{B^{-1} K^{\frac{-1}{2}} u^{\frac{\alpha+1}{2}}}\right]\right|_{u \rightarrow 0} \\
& =-\frac{\imath \omega r_{+}^{\alpha+3}}{2 \pi T l^{\alpha+4}} C_{1}
\end{aligned}
$$

where in the last line Eq.(22) was used.

Now the transverse shear viscosity can be found by using Green-Kubo formula [21],

$$
\eta_{\perp}=\eta_{y y}^{x x}=-\lim _{\omega \rightarrow 0} \frac{1}{\omega} \Im G_{y y}^{x x}(\omega, \overrightarrow{0})=\left(\frac{r_{+}^{3}}{l^{3}}\right) B(u=1) \sqrt{K(u=1)}
$$

Then the ratio of transverse shear viscosity to entropy density is,

$$
\frac{\eta_{\perp}}{s}=\frac{1}{4 \pi} .
$$

The importance of this result is that it is an exact and universal result regardless of details of black-brane solution. There are two kind of shear viscosity in anisotropic black brane solution: transverse and longitudinal. In this paper we calculate transverse shear viscosity. 


\section{Results and discussion}

We prove the universality of the $\frac{\eta_{\perp}}{s}$ for the general anisotropic black brane solution in Horava-Lifshitz gravity. This value for QGP is the same as experimental data. This value is an example for supporting string theory.

\section{Conclusion}

We showed that the lower bound of the ratio $\frac{\eta_{\perp}}{s}$ preserves for the general anisotropic black brane in Horava-Lifshitz gravity. This bound is known as KSS conjecture [21] and considered for strongly interacting systems where reliable theoretical estimate of the viscosity is not available. It tells us that the ratio $\eta / s$ has a lower bound, $\frac{\eta}{s} \geq \frac{\hbar}{4 \pi k_{B}}$, for all relativistic quantum field theories at finite temperature without chemical potential and can be interpreted as the Heisenberg uncertainty principle [19, 21]. The massive term with the Dirichlet boundary condition and regularity on the horizon [25, 26, 27] violate the KSS bound but the massive term with the Petrov-like boundary condition preserve this bound [28]. However, this conjecture violates for higher derivative gravities [29, 30, 31, 32, 33, 34, 35, 36, 37, 38, 39, 40].

Acknowledgment Author would like to thank Shahrokh Parvizi and the referee of INJP for useful comments and suggestions.

\section{References}

[1] J. M. Maldacena, Int. J. Theor. Phys. 38 (1999) 1113 [Adv. Theor. Math. Phys. 2 (1998) 231]

[2] O. Aharony, S. S. Gubser, J. M. Maldacena, H. Ooguri and Y. Oz, Phys. Rept. 323, 183 (2000)

[3] J. Casalderrey-Solana, H. Liu, D. Mateos, K. Rajagopal and U. A. Wiedemann,

[4] Forster D. Hydrodynamic Fluctuations, Broken Symmetry, and Correlation Functions, Reading: Benjamin (1975)

[5] S. Bhattacharyya, V. E. Hubeny, S. Minwalla and M. Rangamani, JHEP 0802, 045 (2008)

[6] M. Rangamani, Class. Quant. Grav. 26, 224003 (2009)

[7] L. D. Landau and E. M. Lifshitz, Fluid Mechanics (Course of Theoretical Physics,Vol. 6). Butterworth-Heinemann, 1965.

[8] J. Bhattacharya, S. Bhattacharyya, S. Minwalla and A. Yarom, JHEP 1405, 147 (2014)

[9] P. Kovtun, J. Phys. A 45 (2012) 473001

[10] D.T. Son, Nuclear Physics B (Proc. Suppl.) 192-193 (2009) 113-118 
[11] Joseph I. Kapusta , Charles Gales; cambridge university press 2006

[12] D. Mateos and D. Trancanelli, JHEP 1107, 054 (2011)

[13] D. Mateos and D. Trancanelli, Phys. Rev. Lett. 107, 101601 (2011)

[14] V. Jahnke, A. S. Misobuchi and D. Trancanelli, JHEP 1501, 122 (2015)

[15] A. Rebhan and D. Steineder, Phys. Rev. Lett. 108, 021601 (2012)

[16] K. A. Mamo, JHEP 1210, 070 (2012)

[17] D. Blas, O. Pujolas and S. Sibiryakov, JHEP 1104, 018 (2011)

[18] R. B. Griffiths, Phys. Rev. Lett. 24, 715 (1970).

[19] D. T. Son and A. O. Starinets, Ann. Rev. Nucl. Part. Sci. 57, 95 (2007)

[20] G. Policastro, D. T. Son and A. O. Starinets, Phys. Rev. Lett. 87, 081601 (2001)

[21] P. Kovtun, D. T. Son and A. O. Starinets, Phys. Rev. Lett. 94, 111601 (2005)

[22] G. Policastro, D. T. Son and A. O. Starinets, JHEP 0209, 043 (2002)

[23] P. Kovtun, D. T. Son and A. O. Starinets, JHEP 0310, 064 (2003)

[24] D.T. Son, Nuclear Physics B (Proc. Suppl.) 192-193 (2009) 113-118.

[25] S. A. Hartnoll, D. M. Ramirez and J. E. Santos, JHEP 1603, 170 (2016)

[26] M. Sadeghi, Eur. Phys. J. C 78, no. 10, 875 (2018)

[27] M. Sadeghi, Mod. Phys. Lett. A 33, no. 37, 1850220 (2018)

[28] W. J. Pan and Y. C. Huang, Phys. Rev. D 94, no. 10, 104029 (2016)

[29] M. Brigante, H. Liu, R. C. Myers, S. Shenker and S. Yaida, Phys. Rev. D 77, 126006 (2008)

[30] M. Brigante, H. Liu, R. C. Myers, S. Shenker and S. Yaida, Phys. Rev. Lett. 100, 191601 (2008)

[31] I. P. Neupane and N. Dadhich, Class. Quant. Grav. 26, 015013 (2009)

[32] M. Sadeghi and S. Parvizi, Class. Quant. Grav. 33, no. 3, 035005 (2016)

[33] S. Parvizi and M. Sadeghi, Eur. Phys. J. C 79, no. 2, 113 (2019)

[34] P. Burikham and N. Poovuttikul, Phys. Rev. D 94, no. 10, 106001 (2016)

[35] X. H. Ge, S. J. Sin, S. F. Wu and G. H. Yang, Phys. Rev. D 80, 104019 (2009)

[36] X. H. Ge, Sci. China Phys. Mech. Astron. 59, no. 3, 630401 (2016)

[37] R. G. Cai, Z. Y. Nie and Y. W. Sun, Phys. Rev. D 78, 126007 (2008)

[38] Y. Z. Li, H. S. Liu and H. Lu, JHEP 1802, 166 (2018)

[39] M. H. Dehghani and M. H. Vahidinia, JHEP 1310, 210 (2013)

[40] Y. L. Wang and X. H. Ge, Phys. Rev. D 94, no. 6, 066007 (2016) 